\title{
George E. Atwood: Der Abgrund des Wahnsinns Psychoanalytische Erkundungen von Erfahrungen seelischer Zerstörung (Giessen: Psychosozial-Verlag, 2017; Original: The Abyss of Madness, 2011)
}

Norbert Wolff(Wetzikon)

Im Prolog von «Der Abgrund des Wahnsinns» beschreibt Atwood als zentrales Ziel dieses Buches, die scharfe Grenze zwischen Wahnsinn und Gesundheit aufzulösen, um die Phänomene schwerer seelischer Störungen wieder in den Kreislauf des menschlich Verstehbaren zurückzubringen. Seinen Standpunkt bezeichnet er als den eines "phänomenologischen Kontextualismus», als einen Weg des Verstehens, der versucht «Psychoanalyse als eine Form phänomenologischer Erkundung neu zu denken» (S. 14).

Atwood hat zusammen mit Donna M. Orange und Robert D. Stolorow eine theoretische Grundlegung der «Intersubjektivität in der Psychoanalyse» formuliert, welche diese als eine «Metatheorie der Psychoanalyse» bezeichnet (Orange, Atwood et al., 2015, S. 11). Diese «untersucht das Feld - zwei Subjektivitäten innerhalb des Systems, das sie erzeugen und aus dem sie hervorgehen - in allen Formen der psychoanalytischen Behandlung. Aufgrund dieses Fokus schliesst die Intersubjektivitätstheorie auch ein kontextualistisches Verständnis von Entwicklung und Pathogenese mit ein» (ebd.). Menschliche Begegnungen werden als relationale Vorgänge beschrieben. Im klinischen Kontext erfolgt dieses wechselseitige Erkennen im intersubjektiven Feld einer Analyse. Die Intersubjektivitätstheorie richtet ihre Aufmerksamkeit konsequent auf die Wechselwirkung zwischen unterschiedlich organisierten Subjektivitäten. Gemäss ihrer Auffassung befindet sich die Therapeutin/der Analytiker mit dem Patienten «in der Situation» (und nicht wie ein teilnehmender Beobachter ausserhalb). Emotionale Erfahrungen werden immer als eingebettet in Beziehungskontexte betrachtet, welche sich wechselseitig konstituieren.

Im Buch «Der Abgrund des Wahnsinns» hat Atwood einige bereits in verschiedenen psychoanalytischen Zeitschriften in den USA veröffentlichte Artikel überarbeitet, durch neue Kapitel ergänzt und so eine umfangreiche Abhandlung über die Entstehung seelischen Leidens und deren Behandlung geschrieben. Aus- 
gehend von lebendig geschilderten Fallgeschichten geht es dem Autor darum, Erfolg und Scheitern therapeutischer Bemühungen darzustellen. Ziel des verstehenden Bemühens ist es, die «innere Wahrheit eines Lebens zu entdecken» (S. 15). Dieses Verstehen geht gemäss Atwood nicht, ohne «im tiefsten Inneren der eigenen persönlichen Existenz» betroffen zu sein (ebd.).

Im ersten Kapitel «Psychotherapie ist eine Humanwissenschaft» beschreibt Atwood, zurückblickend auf 50 Jahre Berufserfahrung, seinen Werdegang als Psychotherapeut anhand von ihn prägenden klinischen Erfahrungen mit PatientInnen. In Fällen von diagnostizierter Psychose und sogenannter «Schizophrenie» erkannte er nicht nur Zeichen einer innerlichen Krankheit, sondern vielmehr verzweifelte Hilferufe und Reaktionen auf anhaltende Erfahrung fortwährenden Verlassen-Werdens, tiefgreifendem Nicht-Verstehen, von verstörender Nicht-Responsivität, vernichtender Nicht-Bestätigung und wiederholten Traumatisierungen.

Im Kapitel (2) «Erkundung des Abgrunds des Wahnsinns» stellt Atwood sein Verständnis von psychischen Zusammenbrüchen (Psychosen) dar. «Wahnsinn ist der Abgrund. Es ist die Erfahrung absoluter Vernichtung» (S. 52). Er betont aufgrund seiner Erfahrung als Psychotherapeut und Psychoanalytiker: «Der Abgrund ist eine Möglichkeit, die jedem menschlichen Leben innewohnt» (S. 53). Sein Behandlungsansatz ist vom Ringen um Verstehen und geduldigem Zuhören geprägt. «Was eine Person im Griff der Vernichtung vor allen Dingen benötigt, ist jemandes Verständnis für den Horror» (S. 56). Atwood äussert sich dezidiert kritisch gegenüber einer Diagnosestellung, insbesondere gegenüber der Klassifikation einer Krankheit als «schizophren», weil diese dem Verständnis nicht dient, sondern vielmehr im Dienst einer Abgrenzung (der Abwehr des Psychotherapeuten) steht. «Eine verdinglichende psychiatrische Diagnose ist das Gegenteil einer benötigten Bestätigung und Spiegelung» (S. 56). Er veranschaulicht sein Erklärungsmodell anhand klinischer Vignetten: «Wahnsinn kommt zustande aus dem Versagen tragender menschlicher Beziehungen. Es entsteht aus desaströsem Trauma, das die ureigene Kapazität in Frage stellt, «Ich bin» zu erleben. (...) Was immer die Details der jeweiligen Entstehungsgeschichte sind, Wahnsinn ist eine menschliche Antwort innerhalb eines menschlichen Kontexts» (S. 65).

Im nächsten Kapitel (3) «Philosophie und Psychotherapie» geht Atwood auf die menschliche Dimension des Selbsterlebens und der Selbstvergewisserung ein, die ein Gegenüber benötigt. Im Zusammenbruch der Psychose zeigt sich der Mangel an Spiegelung, Anerkennung und Bezogenheit in der Entwicklung des Individuums. Häufig findet sich rückblickend eine Eltern-Kind-Beziehung, in der 
das «Sich-selbst-Erleben des Kindes als eigenständige Person eigenen Rechts (...) aufgehoben» ist; «... das rudimentäre «Ich» benötigt ein verstehendes und antwortendes «Du», um eine Chance zu haben, sich selbst zu festigen» (S. 70). Was braucht ein Mensch mit diesem schweren Rucksack in der Therapie? «Mein Ziel wäre es, ihn zu entängstigen und ihm Grund zur Hoffnung zu geben, auf die er und ich aufbauen könnten» (S. 76). Atwood geht auf ethische, erkenntnistheoretische und ästhetische Aspekte ein, die seine therapeutische Haltung beeinflusst haben.

Im Kapitel (4) «Träume und Wahn» versucht Atwood anhand von zwei Fallvignetten bei der Deutung des Traumes «den Kontext der Erfahrung, zu dem der Traum gehört, zu lokalisieren» (S. 99f.) und stösst dabei auf schmerzhafte und extreme Gefühle von Missachtung und Missbrauch. Er geht auf den Zusammenhang zwischen Träumen und Wahnideen ein, welches beide Manifestationen der symbolischen Kapazität der menschlichen Natur darstellen und mit der Sprache und unserer kreativen Fantasietätigkeit verknüpft sind. Er zeigt auf, «wie das Vernichtungserleben eines psychotischen Zustands in Träumen auftaucht und offensichtlich auch in sogenannten Wahnideen» (S. 109).

Im Kapitel (5) «Das Unerträgliche und das Unsagbare» veranschaulicht Atwood die Auswirkungen schwerer Traumatisierungen und geht auf die Schutzmechanismen von Dissoziation und Multiplizität ein. Bei der Behandlung schwer traumatisierter Menschen müsse sich der Analytiker gut mit dem Kind in sich verstehen, damit es eine Chance auf eine erfolgreiche klinische Arbeit mit traumatisierten Patienten geben könne. Die Herausforderung bei der Behandlung von schweren Traumata bestehe darin «das Unerträgliche auszuhalten und das Unsagbare zu sagen» (S. 120). Der erlittene Schmerz könne nicht überwunden werden, sondern es könne lediglich versucht werden, ihn einzubinden, um eine Zukunft in Ganzheit, ohne Verleugnung und Abspaltung der erlittenen Traumata zu leben.

Im Kapitel (6) «Die Tragödie der Selbstzerstörung» geht Atwood auf die dem Menschen innewohnende Möglichkeit zum Selbstmord ein, welche seiner Erfahrung nach «nicht durch Wut und Hass, sondern vielmehr durch Verzweiflung» entsteht (S. 140). Selbstmord versteht er als eine Tür, die jedem menschlichen Wesen zur Verfügung steht, als eine Alternative zu unerträglicher Qual und Hilflosigkeit. «Es ist der klammernde Griff nach Wirkmacht in einer Grube der Verzweiflung» (ebd). In Fallbeispielen beschreibt Atwood die desaströsen Auswirkungen von emotionalem und sexuellem Missbrauch auf die Betroffenen. Im Akt der Selbsttötung komme die Wahrheit einer Erfahrung zum Ausdruck, die geleugnet worden war. 
Im siebten Kapitel «Die dunkle Seite der Melancholie» zeigt Atwood den menschlichen Ursprung der Depression auf. «Depression entsteht aus depressiv machenden Ereignissen» (S. 167). Die depressive Symptomatik könne überwiegend auf eine Kindheit zurückgeführt werden, in der das Kind, um den elterlichen Erwartungen zu genügen, sein wahres Selbst verleugnen und sich anpassen musste. Er beschreibt in Fallbeispielen anschaulich den Prozess und Akt der Selbstaufgabe. Eine Person könne sich jedoch nicht selbst aufgeben, ohne einen hohen Preis dafür zu bezahlen. Der Mensch verliere seine Authentizität und laufe daraufhin Gefahr depressiv zu werden. Einen häufigen Ursprung der Depression sieht Atwood in Erfahrungen des Kindes, dass es die Eltern im Stich lässt, wenn es sich autonom entwickelt und von ihnen fortbewegt. Die Angst über das Zerbrechen der Eltern führt zur Selbstverleugnung, zum Selbstverlust, zur Angst böse und schlecht zu sein.

In Kapitel (8) «Was ist ein Geist?» führt Atwood aus: «Geister sind virtuelle Menschen (...) - sind Möglichkeiten dessen, wer wir sind, wer wir gewesen sind und wer wir hätten werden können» (S. 175). Jede Person trage viele Geister mit sich, lebe mit ihren verlorenen Möglichkeiten, und manchmal können diese sie heimsuchen oder sogar angreifen. Der menschliche Zustand, der bipolare Störung genannt wird, werde nach seiner klinischen Erfahrung häufig durch die plötzliche Ankunft mächtiger Geister aus der Vergangenheit verursacht. Bezugnehmend auf Winnicott führt Atwood aus, dass Manie eine Abwehr gegen die Depression darstelle. Diese Geister haben aber nicht nur ein zerstörerisches Potenzial, sondern befähigen Menschen auch zu kreativen Leistungen, wie der Autor u.a. an den Lebensgeschichten von Vincent Van Gogh und Rainer Maria Rilke aufzeigt.

Im letzten Kapitel (9) «Wahnsinn und Genie postcartesianischer Philosophie» zeigt der Autor am Beispiel der Lebensgeschichte von Kierkegaard, Nietzsche, Wittgenstein und Heidegger auf, «wie die Strukturen unserer privaten Welten in unseren Theorien wiedererscheinen» (S. 185). Atwood zeigt vom intersubjektiven Standpunkt aus auf, wie Verlusterlebnisse in der Kindheit, das erschwerte Ringen um eine eigene Identität, das schwierige Erbe sich von väterlichen Ansprüchen zu emanzipieren und Erfahrungen einer unsicheren eigenen Existenz das theoretische Denken und Werk der vier Philosophen beeinflusst haben.

Dieses Buch zeichnet sich durch eine Fülle an Fallgeschichten von Menschen in schweren existenziellen Krisen und Zuständen des Wahnsinns aus, welche in jahrelanger stationärer und ambulanter Behandlung waren, mit wechselhaften Verläufen, schweren suizidalen Krisen oder wiederholten psychotischen Zusammenbrüchen. In seinen Reflexionen beschreibt Atwood zusammenfassend seinen Behandlungsansatz: Der Schlüssel zum Verstehen und zur Heilung von 
Verrücktheit, von psychotischen Zuständen liege allein in der Macht des menschlichen Verstehens. "We must use our understanding then to discover responses to our patients crisis that will help them refind a personal center and feel included again in the human community" (S. 2). Vielen dieser PatientInnen konnte durch geduldiges Zuhören und das Bemühen um Einfühlung und Verstehen geholfen werden, um aus Isolation, Abschottung und einem wahnhaften Glaubenssystem herauszufinden, um wieder ein aktives Mitglied der menschlichen Gemeinschaft zu werden und ein selbstbestimmtes Leben zu führen. Schwer psychotische und traumatisierte Menschen konnten dank der intensiven psychoanalytischen Behandlung wieder in Kontakt mit ihrem kreativen Potenzial kommen und künstlerisch tätig werden. "That is what is needed in such cases: patience, devotion, and understanding" (2018, S. 8).

«Der Abgrund des Wahnsinns» ist eine inspirierende Lektüre für Fachpersonen, die interessiert an einem verstehenden, intersubjektiven Ansatz bei der Behandlung von schweren seelischen Krisen sind. Der Leser profitiert vom Erfahrungsschatz eines Klinikers, der sich persönlich nicht versteckt und dezidiert kritisch Stellung zu gegenwärtigen Entwicklungen einer biologistischen, objektivierenden Psychiatrie und modularen behavioristischen, evidenzbasierten Behandlungskonzepten nimmt. Als störend empfand ich den besserwisserischen Ton bei der Darstellung von gescheiterten Behandlungen anderer Fachkollegen. Gleichzeitig beschönigt Atwood Erfahrungen persönlichen Scheiterns nicht und berichtet demütig von eigenen Verlusterfahrungen.

Als überzeugend empfand ich Atwoods dezidierten Appell an Psychotherapeuten, sich vom Gegenüber berühren zu lassen, dem Patienten empathisch $\mathrm{zu}$ begegnen und zu verstehen versuchen, was zur Krise geführt hat. Er spricht sich gegen die scharfe Trennung zwischen Beobachter und Beobachtetem aus, sondern betrachtet beide als untrennbar miteinander verbunden, als interdependent. Er vertritt eindrücklich die Originalität und Kraft des psychoanalytischen Behandlungsansatzes: "Psychoanalysis is and always has been an approach to the healing of the wounded soul, and also an exploring of the depths of human existence and human nature" (2018, S. 5).

\section{Literatur}

Orange, D. M., Atwood, G. E. \& Stolorow, R. D. (2015). Intersubjektivität in der Psychoanalyse. Kontextualismus in der psychoanalytischen Praxis. Brandes \& Apsel. Frankfurt a. M.

Atwood, G. E. (2018). Credo and Reflections (http:/ /www.georgeatwood.com/credoand-reflections.html) [15.04.2019]. 